\title{
Mental health of the non-heterosexual population of England
}

\author{
Apu Chakraborty, Sally McManus, Terry S. Brugha, Paul Bebbington and Michael King
}

\section{Background}

There has been little research into the prevalence of mental health problems in lesbian, gay and bisexual (LGB) people in the UK with most work conducted in the USA.

\section{Aims}

To relate the prevalence of mental disorder, self-harm and suicide attempts to sexual orientation in England, and to test whether psychiatric problems were associated with discrimination on grounds of sexuality.

\section{Method}

The Adult Psychiatric Morbidity Survey 2007 ( $n=7403)$ was representative of the population living in private UK households. Standardised questions provided demographic information. Neurotic symptoms, common mental disorders, probable psychosis, suicidality, alcohol and drug dependence and service utilisation were assessed. In addition, detailed information was obtained about aspects of sexual identity and perceived discrimination on these grounds.

\section{Results}

Self-reported identification as non-heterosexual (determined by both orientation and sexual partnership, separately) was associated with unhappiness, neurotic disorders overall, depressive episodes, generalised anxiety disorder, obsessivecompulsive disorder, phobic disorder, probable psychosis, suicidal thoughts and acts, self-harm and alcohol and drug dependence. Mental health-related general practitioner consultations and community care service use over the previous year were also elevated. In the non-heterosexual group, discrimination on the grounds of sexual orientation predicted certain neurotic disorder outcomes, even after adjustment for potentially confounding demographic variables.

\section{Conclusions}

This study corroborates international findings that people of non-heterosexual orientation report elevated levels of mental health problems and service usage, and it lends further support to the suggestion that perceived discrimination may act as a social stressor in the genesis of mental health problems in this population.

\section{Declaration of interest}

None.
There have been few investigations into the prevalence of mental health problems in representative populations of lesbian, gay and bisexual (LGB) people, particularly in Europe. Information about the mental health of LGB people is inconclusive partly because of the difficulty of defining or recruiting samples that are representative of all non-heterosexual people. Obstacles include variation in the definition of sexual orientation, self-harm and mental illness; the cost of achieving representative samples of low-prevalence subgroups; reliance on participants' recall; an unwillingness of people to be open about their sexual orientation; and failure to adjust for pertinent confounding influences such as substance use and personality factors. ${ }^{1,2}$ The majority of studies in this field have been conducted in the USA ${ }^{3-11}$ although some have been carried out in the UK, Continental Europe and Australia. ${ }^{1,12}$ A recent meta-analysis of the prevalence of mental disorder, substance misuse, suicide attempts, suicidal ideation and selfharm in LGB people revealed a doubling of suicide attempts in individuals who are LGB (pooled risk ratio (RR) for lifetime risk $2.47,95 \%$ CI 1.87-3.28). Compared with heterosexual people, the prevalence of depression and anxiety disorders (over a period of 12 months or a lifetime) on meta-analyses was at least 1.5 times higher in individuals who are LGB (RR range 1.54-2.58), and dependence on alcohol and other substance over 12 months was also 1.5 times higher. ${ }^{1}$ Only one small study of LGB mental health, based on pseudo-random sampling has been conducted in the UK but this was limited by small numbers and recruitment of general practice attenders. ${ }^{13}$

Given the high rates of mental health problems in LGB people in North American studies, our aim was to compare the risk for mental disorder, self-harm and suicide attempts in non-heterosexual people with heterosexual people in England, and to assess whether mental distress or diagnosis was associated with discrimination against sexuality. This is a step towards understanding mechanisms of causation and examining the efficacy of prevention efforts. The Adult Psychiatric Morbidity Survey 2007 (APMS 2007) ${ }^{15}$ was primarily conducted by the National Centre for Social Research, with the University of Leicester conducting the second phase of interviews. Compared to earlier surveys in 1993 and 2000 this third survey covered England only and removed the upper age limit to participation. Most particularly, it was the first of the three surveys to contain a question on sexual orientation and same-sex partnerships. Thus, this survey is the first opportunity in England to examine the mental health and well-being of LGB people in a random sample of the general population. The study's aims were first, to determine the prevalence of psychiatric and service-use outcomes by sexual-orientation status in the general population; and second, to examine the extent to which discrimination on the basis of sexual orientation explains any association between sexual identity and psychiatric disorder.

\section{Method}

\section{Fieldwork}

The Adult Psychiatric Morbidity survey series is part of a programme of surveys currently commissioned by the National Health Service (NHS) Information Centre for Health and Social Care, and previously commissioned by the Department of Health. ${ }^{14}$ The sample for APMS 2007 was designed to be representative of the population living in private households (that 
is, people not living in communal establishments) in England. For a detailed description of the survey method please refer to the main report. ${ }^{15}$

\section{Measures}

Standardised questions were asked about age, gender, marital status, ethnic origin and education. Neurotic symptoms and common mental disorders were assessed in the first phase lay interview using the Clinical Interview Schedule Revised (CIS-R). ${ }^{16}$ The CIS-R is an interviewer-administered structured interview schedule covering non-psychotic symptoms in the week prior to interview. It can be used to provide prevalence estimates for five types of common mental disorders according to the ICD-10, ${ }^{16}$ plus a residual category of mixed anxiety and depression, which scores $12+$ on the CIS-R but does not meet the criteria for the other disorders. Probable psychosis was identified from information obtained in a phase one screen that was followed, for a subset of respondents, by a phase two clinical assessment.

Of the 7403 respondents who completed a phase one interview, $313(4 \%)$ met at least one of the psychosis screening criteria, being thereby eligible for a phase two clinical assessment for psychosis. In total $61 \%$ of respondents meeting one or more psychosis criteria at phase one had an assessment using the Schedule for Clinical Assessment in Neuropsychiatry version 2.1 (SCAN). ${ }^{17}$ Because SCAN involves interviewer judgement of whether symptoms are present (as opposed to self-reporting), the interviews were conducted by clinical interviewers from the University of Leicester. The presence of non-organic psychosis, in the year before interview, was established by applying ICD-10 diagnostic algorithms $\left(\mathrm{CATEGO}^{18}\right)$ to the SCAN-generated symptom ratings.

A measure of probable psychosis was generated using the following approach.

(a) Those who screened negative for psychosis at phase one were assumed to be true negatives.

(b) For those who screened positive for psychosis at phase one and had a SCAN assessment, the results of the SCAN were used to determine inclusion in the category.

(c) Those who screened positive for psychosis at phase one but did not have a SCAN assessment (e.g. because of refusal or non-contact) were included in the category if they met two or more psychosis screening criteria.

Suicidal thoughts, attempts and self-harm were assessed from the following three questions: 'have you ever thought of taking your life, even though you would not actually do it?'; 'have you ever made an attempt to take your life, by taking an overdose of tablets or in some other way?; and 'have you ever deliberately harmed yourself in any way but not with the intention of killing yourself?. These were administered in the computer-assisted self-completion section of the interview, via a laptop computer.

Alcohol use was assessed with the Alcohol Use Disorders Identification Test (AUDIT). ${ }^{19}$ The alcohol dependence of those who scored 10 or more on AUDIT was assessed using the community version of the Severity of Alcohol Dependence Questionnaire (SADQ-C) ${ }^{20}$ The SADQ-C consists of 20 items covering a range of dependence symptoms, with the 6 months before the interview as the reference period. Answers to all questions are scored from 0 to 3 , and summed to give a total score ranging from 0 to 60 . A score of four or more was used to indicate presence of alcohol dependence in the past 6 months.
Questions about drug dependence were asked in the computer-assisted self-completion section. For each of eight drug types (cannabis, amphetamines, crack, cocaine, ecstasy, tranquillisers, opiates and volatile substances), reported use in the past year was followed by five questions based on the Diagnostic Interview Schedule ${ }^{21}$ and designed to assess drug dependence in the past year.

Respondents were asked about consultations with their general practitioner (GP) or family doctor in the past year, and whether these had been for a mental or emotional problem, for a physical problem or for both. The figures presented here relate to GP contacts in relation to mental and emotional problems. Respondents were also asked about community care services used in the past year. These included contact with a psychiatrist, psychologist, community nurse services, a social worker, self-help or support groups, home helps, outreach workers and a community day care centre.

Happiness was measured with a single question: 'taking all things together, how would you say you are these days - very happy, fairly happy, or not too happy?' This measure has been used in a number of studies of happiness and well-being. ${ }^{22}$ Experience of discrimination in the past year was asked about in the computer-assisted self-completion section of the interview. A series of questions asked whether the respondent had been unfairly treated on the basis of their age, gender, religion, ethnicity, health or disability, mental health or sexual orientation. No context for this discrimination was specified. The question on unfair treatment because of sexual identity was the variable used in these analyses.

Immediately following the discrimination section in the selfcompletion questionnaire were questions on self-perceived sexual identity (using a five-point response scale) and on the gender of sexual partners. Because of the relatively small number of respondents reporting an identity other than entirely heterosexual, these were grouped together to form a binary variable of 'heterosexual' and 'non-heterosexual'. The 'non-heterosexual' category included respondents describing their sexual identity as 'mostly heterosexual' and as 'other' as well as gay, lesbian and bisexual. A similar binary variable was derived for partnership, with all respondents reporting any same-gender partners being grouped together in an inclusive category (Appendix).

\section{Statistical analysis}

The survey data were weighted to represent the national population, taking account of non-response, size of household, characteristics of non-responding households and the profile of age and gender within the government office region.

The 'survey' commands in STATA 10.0 for Windows were used, which allow for the use of clustered data modified by probability weights. The variables studied are described using actual numbers and weighted proportions. Percentage prevalence of various mental health conditions was calculated on the basis of sexual identity and partnership status. Bivariate statistics were first used to determine whether there were significant differences in mental health between people of differing sexual orientation. Next, multivariate regression was performed to determine whether sexual identity or partnership status predicted: mental disorder, contact with psychiatric services or contact with the GP in relation to psychiatric problems, after adjustment for sociodemographic variables. Finally the prevalence of discrimination in relation to sexuality was determined, and analysis was performed to see whether discrimination on the basis of sexual orientation predicted neurotic disorder or probable psychosis within the non-heterosexual group. 


\section{Results}

Most of the psychiatric disorders and behaviours assessed had significantly elevated prevalence among people reporting non-heterosexual orientation and those reporting they had had partners of the same gender compared with the rest of the population (Table 1). With regard to sexual orientation, unhappiness, the prevalence of any neurotic disorder in the past week, depressive episodes in the past week, generalised anxiety disorder, obsessive-compulsive disorder, phobic disorder, lifetime suicidal thoughts, lifetime suicide attempts, lifetime self-harm, probable psychosis, drug dependence in the past year and alcohol dependence in the past 6 months were significantly more prevalent in people who self-identified as non-heterosexual rather than heterosexual. The pattern of results was similar for the sexual partnership question (Appendix), except for drug and alcohol dependence, which ceased to be significant.

We then compared non-heterosexual and heterosexual people on prevalence of psychiatric disorder and use of health services, while adjusting for the key sociodemographic variables gender, age, ethnicity and holding an educational qualification (Table 2). Only those variables that were significant were retained in each of the final models. For the group that was non-heterosexual in terms of sexual orientation, the adjusted odds ratios (OR) for neurotic disorder overall $(\mathrm{OR}=1.47)$, depressive episode $(\mathrm{OR}=1.80)$, obsessive-compulsive disorder $(\mathrm{OR}=2.24)$, phobic

\begin{tabular}{|c|c|c|c|c|c|c|}
\hline & \multicolumn{3}{|c|}{ Sexual orientation } & \multicolumn{3}{|c|}{ Sexual partner } \\
\hline & $\begin{array}{l}\text { Heterosexual, \% } \\
\qquad(n=6811)\end{array}$ & $\begin{array}{l}\text { Non-heterosexual, \% } \\
\qquad(n=650)\end{array}$ & $P$ & $\begin{array}{c}\text { Only opposite } \\
\text { gender, } \%(n=6794)\end{array}$ & $\begin{array}{c}\text { Any same gender, } \\
\%(n=667)\end{array}$ & $P$ \\
\hline Fairly or very happy & 40.1 & 30.3 & $<0.0001$ & 39.9 & 32.5 & 0.0007 \\
\hline Any neurotic disorder in past week ${ }^{\mathrm{b}}$ & 15.4 & 22.2 & $<0.0001$ & 15.5 & 21.4 & $<0.0001$ \\
\hline Depressive episode in past week ${ }^{\mathrm{b}}$ & 2.1 & 4.1 & 0.0037 & 2.1 & 4.0 & 0.0024 \\
\hline Generalised anxiety disorder $^{\mathrm{b}}$ & 4.2 & 6.3 & 0.014 & 4.2 & 6.0 & 0.027 \\
\hline Obsessive-compulsive disorder ${ }^{\mathrm{b}}$ & 1.0 & 2.6 & 0.017 & 0.9 & 2.6 & 0.0083 \\
\hline Phobic disorder ${ }^{c}$ & 1.3 & 2.8 & 0.019 & 1.3 & 2.5 & 0.0064 \\
\hline Probable psychosis ${ }^{\mathrm{c}}$ & 0.38 & 1.4 & 0.0003 & 0.39 & 1.2 & 0.0038 \\
\hline Drug dependence in past year & 3.1 & 6.1 & 0.0009 & 3.2 & 4.5 & $0.15^{\mathrm{a}}$ \\
\hline Alcohol dependence in past six months ${ }^{d}$ & 5.4 & 10.4 & $<0.0001$ & 5.7 & 7.3 & $0.058^{a}$ \\
\hline Suicidal thoughts, lifetime & 16.1 & 20.6 & 0.037 & 15.5 & 24.5 & $<0.0001$ \\
\hline Suicide attempts, lifetime & 5.3 & 8.9 & 0.0037 & 5.1 & 9.2 & 0.0001 \\
\hline Self-harm, lifetime & 4.6 & 8.6 & 0.0047 & 4.2 & 10.5 & $<0.0001$ \\
\hline
\end{tabular}

\begin{tabular}{|c|c|c|c|c|}
\hline & \multicolumn{2}{|c|}{ Non-heterosexual orientation } & \multicolumn{2}{|c|}{ Non-heterosexual partnered } \\
\hline & Unadjusted OR (95\% Cl) & Adjusted OR (95\% Cl) & Unadjusted OR (95\% Cl) & Adjusted OR (95\% Cl) \\
\hline Fairly or very happy & $0.65(0.53-0.80)$ & $0.67^{\mathrm{a}}(0.55-0.82)$ & $0.73(0.60-0.87)$ & $0.75^{\mathrm{a}}(0.62-0.90)$ \\
\hline Any neurotic disorder & $1.56(1.26-1.93)$ & $1.47^{\mathrm{b}}(1.18-1.82)$ & $1.48(1.19-1.86)$ & $1.40^{\mathrm{b}}(1.11-1.77)$ \\
\hline Depressive episode & $1.95(1.24-3.09)$ & $1.80^{\mathrm{b}}(1.13-2.87)$ & $1.88(1.25-2.84)$ & $1.77^{\mathrm{b}}(1.15-2.70)$ \\
\hline Generalised anxiety disorder & $1.56(1.09-2.24)$ & $1.49^{\mathrm{C}}(1.03-2.15)$ & $1.47(1.04-2.08)$ & $1.46^{\mathrm{C}}(1.02-2.09)$ \\
\hline Obsessive-compulsive disorder & $2.67(1.43-4.98)$ & $2.24^{d}(1.18-4.27)$ & $2.81(1.26-6.25)$ & $2.47^{\mathrm{e}}(1.12-5.45)$ \\
\hline Phobic disorder & $2.11(1.22-3.62)$ & $1.91^{\mathrm{b}}(1.07-3.39)$ & $1.93(1.19-3.12)$ & $1.77^{\mathrm{b}}(1.04-3.02)$ \\
\hline Probable psychosis & $3.75(1.76-8.00)$ & - & 3.09 (1.39-6.89) & - \\
\hline Drug dependence & $2.05(1.34-3.15)$ & $1.70^{\mathrm{b}}(1.06-2.73)$ & $1.42(0.87-2.30)$ & - \\
\hline Alcohol dependence & $2.04(1.46-2.86)$ & $2.05^{f}(1.45-2.90)$ & $1.31(0.91-1.90)$ & - \\
\hline Suicidal thoughts, lifetime & $1.78(1.43-2.23)$ & $1.85^{\dagger}(1.49-2.30)$ & $1.77(1.42-2.21)$ & $1.84^{f}(1.48-2.30)$ \\
\hline Suicide attempts, lifetime & $2.31(1.70-3.14)$ & $2.21^{\mathrm{c}}(1.61-3.05)$ & $1.87(1.36-2.56)$ & $1.78^{\mathrm{c}}(1.27-2.49)$ \\
\hline Self-harm, lifetime & $3.11(2.16-4.48)$ & $2.82^{\mathrm{g}}(1.94-4.10)$ & $2.69(1.96-3.68)$ & $2.26^{g}(1.62-3.15)$ \\
\hline $\begin{array}{l}\text { Consultations with GP for psychiatric problems, } \\
\text { past year }\end{array}$ & $1.50(1.19-1.90)$ & $1.46^{\mathrm{b}}(1.14-1.86)$ & $1.36(1.07-1.74)$ & $1.35^{\mathrm{b}}(1.04-1.74)$ \\
\hline Community care, past year & $1.95(1.48-2.56)$ & $1.94^{\mathrm{a}}(1.48-2.55)$ & $1.88(1.42-2.50)$ & $1.88^{\mathrm{a}}(1.42-2.49)$ \\
\hline $\begin{array}{l}\text { OR, odds ratio; GP, general practitioner. } \\
\text { a. Adjusted for gender, ethnicity and education. } \\
\text { b. Adjusted for gender, age and education. } \\
\text { C. Adjusted for gender, age, ethnicity and education. } \\
\text { d. Adjusted for age and education. } \\
\text { e. Adjusted for age. } \\
\text { f. Adjusted for gender, age and ethnicity. } \\
\text { g. Adjusted for age, ethnicity and education. }\end{array}$ & & & & \\
\hline
\end{tabular}


disorder $(\mathrm{OR}=1.91)$, probable psychosis $(\mathrm{OR}=3.75)$, drug dependence $(\mathrm{OR}=1.70)$, alcohol dependence $(\mathrm{OR}=2.05)$, as well as lifetime suicidal thoughts $(\mathrm{OR}=1.85)$, suicide attempts $(\mathrm{OR}=2.21)$ and self-harm $(\mathrm{OR}=2.82)$ were all elevated, whereas that for happiness $(\mathrm{OR}=0.67)$ was reduced. Once again the results for the non-heterosexual group in terms of sexual partnerships were similar, being little affected by the adjustment. However, drug and alcohol dependence ceased to be significant.

These raised prevalences appear also to be reflected in service use: consultations with the GP for reasons of mental health $(\mathrm{OR}=1.46)$ and psychiatric community care contacts for the previous year $(\mathrm{OR}=1.94)$ were significantly higher in the nonheterosexual than the heterosexual group, according to both sexual orientation (figures quoted) and to partnership.

Discrimination on grounds of sexuality was more commonly reported in the non-heterosexual than the heterosexual group (Table 3) and this was statistically significant for both sexual orientation and partnership. A total of $4.9 \%$ of non-heterosexual people reported having experienced discrimination because of their sexual orientation in the past 12 months, compared with $1.6 \%$ in the heterosexual group.

Finally, Table 4 reports the effect of discrimination on the basis of sexual orientation upon psychiatric disorder outcomes. Unfair treatment perceived to be due to sexual orientation was investigated to see whether it predicted psychiatric disorder; the association was adjusted for the appropriate sociodemographic confounders. The adjusted odds ratios for occurrence of any neurotic disorder $(\mathrm{OR}=4.24)$ and mixed anxiety/depressive disorder $(\mathrm{OR}=2.60)$ were significantly elevated, with sex discrimination as a primary independent variable. The pattern was similar for the sexual partnership question, except for mixed anxiety/depressive disorder, which lost significance.

\section{Discussion}

\section{Main findings}

This is the first probability sample household survey within the UK to evaluate the mental health and service use of people according to their sexual orientation. Two questions were used to address sexual orientation and sexual partnership, in order to measure the prevalence of same-sex orientation and behaviour in the English general population.
The impact of enquiry format on reporting will be described in greater detail in a separate article (details available from the author on request). In brief, each question had two versions (Appendix): version A of the sexual-orientation question used 'homosexual', version B used 'gay or lesbian'. Version A of the sexual-partnership question required respondents who had male and female partners to say which was predominant, whereas version B had a midpoint response option: 'about equally with men and women'. Participants were randomised to a version. Overall, approximately $8 \%$ reported that they were not entirely heterosexual. The question using the terms 'gay or lesbian' elicited higher reporting of non-heterosexual orientation than the question using the word 'homosexual'. A larger proportion of men and women reported entirely heterosexual partnerships in response to version $\mathrm{A}$ of the partnership question than in response to version B. This would seem to justify the separate analyses for sexual partnership and orientation. Rate of refusal to answer the sexual-orientation questions was very small (a fraction of a percentage point), which makes it inappropriate to draw conclusions about those who refused as a discrete group. Certainly, no significant trends were apparent in non-responders to the two versions of question.

Self-reported identification as non-heterosexual was associated with a significantly elevated prevalence of unhappiness, any neurotic disorder, depressive episodes in the previous week, generalised anxiety disorder, obsessive-compulsive disorder, phobic disorder, probable psychosis, suicidal thoughts and acts and self-harm, alcohol dependence and drug dependence. All of these associations remained significant after adjustment for sociodemographic variables. Finally, discrimination on grounds of sexuality was reported significantly more by the non-heterosexual than the heterosexual group.

\section{Limitations}

The study's limitations include tentative inferences being made about causality from a cross-sectional survey, and the issue of non-response bias when investigating a potentially sensitive area. With regard to deductions about causality, the problem is that one infers past exposure (e.g. discrimination) and outcome (e.g. psychiatric disorder) from concurrent measurements. As a result, the causal sequence can be difficult to assess: did the 'outcome' influence the measured exposure level, or did the exposure affect the outcome? However, it confirms work mainly conducted in

\begin{tabular}{|c|c|c|c|c|c|c|}
\hline Sex discrimination & $\begin{array}{c}\text { Non-heterosexual } \\
\text { orientation, \% }\end{array}$ & $\begin{array}{l}\text { Heterosexual } \\
\text { orientation, \% }\end{array}$ & $P$ & $\begin{array}{l}\text { Non-heterosexual } \\
\text { partnered, \% }\end{array}$ & $\begin{array}{l}\text { Heterosexual } \\
\text { partnered, \% }\end{array}$ & $P$ \\
\hline Yes & 4.9 & 1.6 & & 3.2 & 1.7 & \\
\hline No & 95.0 & 98.4 & $<0.0001$ & 96.8 & 98.3 & 0.0217 \\
\hline
\end{tabular}

Table 4 Discrimination on the basis of sexual orientation as a putative predictor of psychiatric disorder (adjusted for significant confounders

\begin{tabular}{|c|c|c|c|c|}
\hline & \multicolumn{2}{|c|}{ Non-heterosexual orientation } & \multicolumn{2}{|c|}{ Non-heterosexual partnered } \\
\hline & Unadjusted OR ( $95 \% \mathrm{Cl})$ & Adjusted OR (95\% Cl) & Unadjusted OR (95\% Cl) & Adjusted OR $(95 \% \mathrm{Cl})$ \\
\hline Any neurotic disorder & $5.24(2.46-11.14)$ & $4.24^{\mathrm{a}}(1.93-9.28)$ & $4.25(1.86-9.68)$ & $4.11^{b}(1.79-9.41)$ \\
\hline Depressive episode & $6.95(2.74-17.67)$ & - & $7.91(2.90-21.58)$ & - \\
\hline Mixed anxiety/depressive disorder & $2.77(1.19-6.45)$ & $2.60^{\mathrm{b}}(1.10-6.13)$ & $1.59(0.53-4.77)$ & - \\
\hline Obsessive-compulsive disorder & $6.15(1.62-23.34)$ & - & 11.49 (3.37-39.23) & - \\
\hline
\end{tabular}


North America as well as in Holland ${ }^{8}$ that suggests non-heterosexual individuals are at higher risk of mental disorder, suicidal ideation, substance misuse and self-harm than heterosexual people. ${ }^{1}$ It also confirms the results of earlier UK research that employed a 'snowball' sampling technique: that gay men were more likely than heterosexual men to score above threshold on the Clinical Interview Schedule, indicating greater levels of psychological distress, as were lesbians compared with heterosexual women. ${ }^{23}$

\section{Findings from other research}

Mental health-related GP consultations and community-care service use were also elevated in the non-heterosexual population for the previous year in comparison with the heterosexual population. This may be a reflection of the increased level of mental health problems, prompting the health-seeking behaviour. This too is underresearched; a US review of mental health information on gay and bisexual men who had recently attended a US community health clinic found that they most frequently presented with depression, anxiety and relationship issues. ${ }^{24}$ Grella and colleagues found that compared with individuals without a diagnosed mental health or substance use disorder, those with a disorder were more likely to receive treatment. ${ }^{25}$ After controlling for both the presence of disorder and other factors, lesbians and bisexual women were most likely and heterosexual men were the least likely to receive treatment. Moreover, a considerable proportion of those belonging to a sexual minority group without any diagnosable disorder, particularly lesbians and bisexual women, also reported receiving treatment.

The finding that non-heterosexual people are more likely than heterosexuals to seek help for mental health problems or distress is interesting, given that the responses of mental health professionals are not always optimal or appropriate. ${ }^{26,27}$ However, this increased service use may simply reflect an increased need; either way, a service response would be required to serve the mental health needs of this group.

The increased prevalence of discrimination reported by this non-heterosexual sample on the grounds of sexual orientation was expected but the absolute level of discrimination was comparatively low. This result, however, may have been mediated by the wording of the question about discrimination, or it may indicate an adaptation to chronic discrimination. This is echoed by the National Survey of Midlife Development in the USA, which found that homosexual and bisexual individuals $(n=73)$ more frequently than heterosexual people reported both lifetime and day-to-day experiences of discrimination on the basis of sexuality. ${ }^{28}$ Perceived discrimination was associated with both harmful effects on quality of life and indicators of psychiatric morbidity in the total sample. Writers have proffered a conceptual framework for understanding this in terms of minority stress explaining that stigma, prejudice and discrimination create a hostile and stressful social environment that causes mental health problems. ${ }^{29}$ Their model describes stress processes, including the experience of prejudice events, expectations of rejection, hiding and concealing, internalised homophobia and ameliorative coping processes. What was also an interesting and unexpected finding was that the heterosexual group also reported discrimination on the basis of sexual orientation, albeit at a reduced prevalence. This may reflect the existence of prejudice against heterosexuals ${ }^{30}$ that has been anecdotally reported in gay majority environments. It is also possible that effeminate heterosexual men or masculine heterosexual women may have suffered prejudice from stereotyping in which they are assumed to be gay or lesbian.

We found that in the non-heterosexual group, discrimination on the grounds of sexual orientation predicted neurotic disorder outcomes, even after adjustment for the appropriate confounding demographic variables. The association between discrimination and obsessive-compulsive disorder was unexpected. However, obsessional and compulsive symptoms were reported, in a recent systematic review, to be associated with self-reported racial discrimination on all occasions in different studies. ${ }^{31}$ One explanation may be that discriminatory events are recalled more readily in those with an obsessive tendency, who may ruminate about the event. It may be regarded as surprising that in Table 4 there was no positive association found between discrimination on the basis of sexual orientation and psychosis, despite the large prevalence differential according to sexual orientation/partnership for psychosis reported in Table 1. This may be explained by the survey inquiring into discrimination over the previous 12 months; one would expect a more sustained, longer-term exposure to discrimination to be predictive of psychosis. Conclusions about causality need to be tentative with a cross-sectional survey; however, this lends some support to the perceived discrimination-social stressor hypothesis for genesis of mental health problems in the non-heterosexual population of England.

These elevated levels of psychiatric problems in nonheterosexual people are very worrying and call not only for a response by professionals in primary care and mental health services but also efforts at prevention.

\section{Apu Chakraborty, BA, MA, MB BS, MRCPsych, MSC, DLSHTM, PhD, Department of Mental Health Sciences, University College London, London; Sally McManus, MSc, National Centre for Social Research, London; Terry S. Brugha, FRCPsych, Department of Health Sciences, University of Leicester, Leicester; Paul Bebbington MA, MPhil, PhD, FRCP, FRCPsych, Michael King, MD, PhD, Department of Mental Health Sciences, Royal Free and University College Medical School, London, UK \\ Correspondence: Apu Chakraborty, Department of Mental Health Sciences, University College London, Royal Free Campus, Rowland Hill Street, London NW3 2PF, UK. Email: rejuatc@ucl.ac.uk \\ First received 9 May 2010, final revision 13 Oct 2010, accepted 3 Nov 2010}

\section{Appendix}

\section{Sexual orientation and partnerships}

\section{Version A}

Which statement best describes your sexual orientation? This means sexual feelings, whether or not you have had any sexual partners.

1 Entirely heterosexual (attracted to persons of the opposite of sex).

2 Mostly heterosexual, some homosexual feelings.

3 Bisexual (equally attracted to men and women).

4 Mostly homosexual, some heterosexual feelings.

5 Entirely homosexual (attracted to persons of the same sex).

6 Other.

Have your sexual partners been ...

1 only opposite sex

2 mainly opposite sex but some same-sex partners

3 mainly same sex but some opposite-sex partners

4 only same sex

5 or, I have not had a sexual partner.

\section{Version B}

Please choose the answer below that best describes how you think of yourself .

1 completely heterosexual

2 mainly heterosexual

3 bisexual

4 mainly gay or lesbian 


\section{5 completely gay or lesbian \\ 6 Other.}

Sexual experience is any kind of contact with another person that you felt was sexual (it could be just kissing or touching, or intercourse, or any other form of sex). Has your sexual experience been . . .

1 only with (women/men) or a (woman/man), never with a (man/woman)

2 more often with (women/men), and at least once with a (man/woman)

3 about equally often with (women/men) and (men/women)

4 more often with (men/women), and at least once with a (woman/man)

5 only with (men/women) or a (man/woman), never with a (woman/man)

6 I have never had any sexual experience with anyone at all.

\section{References}

1 King M, Semlyen J, Tai SS, Killaspy H, Osborn D, Popelyuk D, et al. A systematic review of mental disorder, suicide, and deliberate self harm in lesbian, gay and bisexual people. BMC Psychiatry 2008; 8: 70.

2 McManus S. Sexual Orientation Research: A Review of Methodological Approaches. Scottish Executive, 2003.

3 Cochran SD, Mays VM. Relation between psychiatric syndromes and behaviorally defined sexual orientation in a sample of the US population. Am J Epidemiol 2000; 151: 516-23.

4 Cochran SD, Mays VM. Lifetime prevalence of suicidal symptoms and affective disorders among men reporting same-sex sexual partners: results from the NHANES III. Am J Public Health 2000; 90: 573-8.

5 Cochran SD, Keenan C, Schober C, Mays VM. Estimates of alcohol use and clinical treatment needs among homosexually active men and women in the US population. J Consult Clin Psychol 2000; 68: 1062-71.

6 Gilman SE, Cochran SD, Mays VM, Hughes M, Ostrow D, Kessler RC. Risk of psychiatric disorders among individuals reporting same-sex sexual partners in the National Comorbidity Survey. Am J Public Health 2001; 91: 933-9.

7 Cochran SD, Sullivan JG, Mays VM. Prevalence of psychiatric disorders, psychological distress, and treatment utilization among lesbian, gay, and bisexual individuals in a sample of the US population. J Consult Clin Psychol, in press.

8 Sandfort TGM, de Graaf R, Bijl RV, Schnabel P. Same-sex sexual behavior and psychiatric disorders: findings from the Netherlands Mental Health Survey and Incidence Study (NEMESIS). Arch Gen Psychiatry 2001; 58: 85-91.

9 Remafedi G, French S, Story M, Resnick MD, Blum R. The relationship between suicide risk and sexual orientation: results of a population-based study. Am J Public Health 1998; 88: 57-60.

10 Garofalo R, Wolf RC, Wissow LS, Woods ER, Goodman E. Sexual orientation and risk of suicide attempts among a representative sample of youth. Arch Pediatr Adolesc Med 1999; 153: 487-93.

11 Faulkner $\mathrm{AH}$, Cranston $\mathrm{K}$. Correlates of same-sex sexual behavior in a random sample of Massachusetts high school students. Am J Public Health 1998; 88: 262-6.

12 Warner J, McKeown E, Griffin M, Johnson K, Ramsay A, Cort C, et al. Rates and predictors of mental illness in gay men, lesbians and bisexual men and women: results from a survey based in England and Wales. Br J Psychiatry 2004; 185: 479-85.
13 King M, Nazareth I. The health of people classified as lesbian, gay and bisexual attending family practitioners in London: a controlled study. BMC Public Health 2006; 6: 127.

14 Jenkins $\mathrm{R}$, Meltzer $\mathrm{H}$, Bebbington $\mathrm{P}$, Brugha TS, Farrell M, McManus $\mathrm{S}$, et al. The British Mental Health Survey Programme: achievements and latest findings. Soc Psychiat Epidemiol 2009; 44: 899-904.

15 McManus S, Meltzer $\mathrm{H}$, Brugha $\mathrm{T}$, Bebbington $\mathrm{P}$, Jenkins R. Adult Psychiatric Morbidity in England, 2007: Results of a Household Survey. NHS Information Centre, 2009.

16 Lewis G, Pelosi AJ, Araya R, Dunn G. Measuring psychiatric disorder in the community; a standardised assessment for use by lay interviewers. Psychol Med 1992; 22: 465-86.

17 Wing JK, Babor T, Brugha T, Burke J, Cooper JE, Giel R, et al. SCAN: Schedules for Clinical Assessment in Neuropsychiatry. Arch Gen Psychiatry 1990; 47: 589-93.

18 Wing JK, Cooper JE, Sartorius N. The Description and Classification of PSychiatric Symptoms: An Instruction Manual for the PSE and CATEGO System. Cambridge University Press, 1974.

19 Saunders JB, Aasland OG, Babor TF, Dela Fuente JR, Grant M. Development of the Alcohol Use Disorders Identification Test (AUDIT): WHO collaborative project on early detection of persons with harmful alcohol consumption, part II. Addiction 1993; 88: 791-804.

20 Stockwell T, Sitharan T, McGrath D, Lang E. The measurement of alcoho dependence and impaired control in community samples. Addiction, 1994; 89: $167-74$.

21 Malgady RG, Rogler LH, Tryon WW. Issues of validity in the Diagnostic Interview Schedule. J Psychiatr Res 1992; 26: 59-67.

22 Ouweneel P, veenhoven R. Cross-national differences in happiness. In Contemporary Issues in Cross-Cultural Psychology (eds N Bleichrodt, PJ Drenth). Swets \& Zeitlinger, 1991.

23 King M, McKeown W, Warner J, Ramsay A, Johnson K, Cort C, et al. Mental health and quality of life of gay men and lesbians in England and Wales: controlled, cross-sectional study. Br J Psychiatry 2003; 183: 552-8.

24 Berg MB, Mimiaga MJ, Safren SA. Mental health concerns of gay and bisexual men seeking mental health services. J Homosex 2008; 54: 293-306.

25 Grella C, Greenwell L, Maysand V, Cochran S. Influence of gender, sexual orientation, and need on treatment utilization for substance use and mental disorders: findings from the California Quality of Life Survey. BMC Psychiatry 2009; 9: 52.

26 King M, Semlyen J, Killaspy H, Nazareth I, Osborn D. A Systematic Review of Research on Counselling and Psychotherapy for Lesbian, Gay, Bisexual and Transgender People. British Association for Counselling and Psychotherapy, 2007.

27 Bartlett A, Smith G, King M. The response of mental health professionals to clients seeking help to change or redirect same-sex sexual orientation. BMC Psychiatry 2009: 9: 11

28 Mays V M, Cochran SD. Mental health correlates of perceived discrimination among lesbian, gay, and bisexual adults in the United States. Am J Public Health 2001; 91: 1869-76.

29 Meyer $\mathrm{IH}$. Prejudice, social stress, and mental health in lesbian, gay, and bisexual populations: conceptual issues and research evidence. Psychol Bull 2003; 129: 674-97.

30 Francoeur RT. The Complete Dictionary of Sexuality. Continuum, 1995.

31 Paradies Y. A systematic review of empirical research on self-reported racism and health. Int J Epidemiol 2006; 35: 888-901. 